\title{
COMPETENCIAS HABILITANTES: UN APORTE PARA EL REFORZAMIENTO DE LAS TRAYECTORIAS FORMATIVAS UNIVERSITARIAS
}

\author{
Marisol Latorre, Paloma Aravena, Pedro Milos, Miguel \\ García $^{1}$
}

\begin{abstract}
RESUMEN
En el contexto de un sistema universitario en expansión, se reflexiona sobre los cambios en los perfiles de ingreso de los estudiantes y las crecientes exigencias al momento del egreso. Ante el dilema de optar entre mayores filtros de entrada o evaluar los resultados finales de la formación, se propone poner el énfasis en las trayectorias formativas concebidas como factor clave para asegurar la calidad y oportunidad de los aprendizajes conducentes a los perfiles de egreso. Se introduce la noción de competencia habilitante en la reflexión y diseño curricular, entendidas como instancias de integración de aprendizajes a lograr al término de los dos primeros años de su formación, con el fin de enfrentar con éxito los años de formación profesional.
\end{abstract}

Palabras clave: competencias habilitantes, formación profesional, educación superior, calidad de la educación, educación basada en el logro de capacidades, educación, currículum.

\section{ENABLING COMPETENCIES: AN INPUT FOR STRENGTHENING FORMATIVE UNIVERSITY YEARS}

\begin{abstract}
In the context of an expanding university system, changes in the graduate profile and the growing demands at graduation time have become a focus of deep reflection. In front of the possibility to apply more entrance filters or evaluate secondary education final results, the proposal is to focus on the formative trajectories conceived as the crucial factor to guarantee quality and relevance of the learning outcomes conducive to the graduate profile. The notion of Enabling Competencies is introduced in the reflection and curriculum design in order to favour the integration of learning outcomes to be achieved at the end of the first two years of university so students can face successfully their professional education.
\end{abstract}

Key words: enabling competencies, professional education, university education, quality of education, education based on skills, education, curriculum.

1 Dirección de Docencia, Facultad de Educación, Universidad Alberto Hurtado, Chile. Contacto:mlatorre@uahurtado.cl 


\section{COMPETENCIAS HABILITANTES: UN APORTE PARA EL REFORZAMIENTO DE LAS TRAYECTORIAS FORMATIVAS UNIVERSITARIAS}

\section{Antecedentes}

Una de las principales tensiones de la educación superior, en la actualidad, dice relación con la posibilidad real de que los estudiantes logren a lo largo de su formación (en general entre cuatro y cinco años) las competencias profesionales que se encuentran contenidas en el perfil de egreso de sus carreras. Los argumentos que aluden a las dificultades de alcanzar este logro son muchos, entre otros: insuficiencia del tiempo disponible, las características de los estudiantes que ingresan, las debilidades de las mallas curriculares y la sobrecarga de los planes de estudio.

Por otra parte, la perspectiva de desarrollo de competencias se ha venido instalando de manera progresiva en el área. Ello ha implicado una revisión y, muchas veces, una reformulación de la lógica de los diseños y mallas curriculares, así como de su implementación, promoviendo una concepción de perfiles de egreso fuertemente centrados en los aprendizajes a lograr.

Los dos puntos anteriores, sumados a las demandas de los sistemas de aseguramiento de la calidad, configuran un escenario crecientemente exigente para las carreras que deben "asegurar" la existencia de ciertas condiciones para el logro del compromiso formativo. En efecto, las carreras enfrentan la necesidad de ofrecer a sus estudiantes oportunidades sustantivas de aprendizaje que garanticen el desarrollo de "buenas trayectorias" académico-formativas. En este contexto, se estima que los niveles de desarrollo de las competencias llamadas básicas, por parte de los estudiantes, son un factor clave a considerar, tanto al inicio como para el desarrollo exitoso de dichas trayectorias. 
Este factor se torna particularmente relevante tratándose de alumnos que evidencian bajos niveles de logro en dichas competencias (expresados, por ejemplo, en sus puntajes de la Prueba de Selección Universitaria (PSU). A este respecto, la tendencia ha sido a reconocer la existencia de estos déficits, al inicio de las trayectorias formativas e implementar, en los primeros años de carrera, acciones "remediales". Sin atender, no obstante, a la posibilidad de abordar y potenciar las relaciones entre el fortalecimiento de dichas competencias y los requerimientos específicos de las distintas formaciones.

La Universidad Alberto Hurtado (UAH), desde el año 2006, ha desarrollado una política de admisión consistente en impulsar el aumento progresivo de los puntajes de ingreso de los nuevos estudiantes y profundizar en el diagnóstico de las habilidades de entrada de cada nueva cohorte. Lo primero se entiende el marco de las orientaciones generales de las políticas para educación superior y, en particular, del desarrollo que ha tenido el sistema universitario, donde contar con estudiantes de puntajes elevados es percibido como un componente más de una oferta académica de calidad. Lo segundo, en cambio, responde a una iniciativa y un interés local, vinculados a la misión institucional de ser una alternativa de formación profesional de calidad que aporte al logro de mayores niveles de equidad social. En este contexto, disponer de una mejor caracterización de los estudiantes que ingresan -por medio de la aplicación de pruebas específicas en áreas básicas, como se viene haciendo desde 2006- permite, por una parte, cualificar los datos de los perfiles de ingreso tradicionales y, por otra, identificar una línea de base útil para la instalación de los procesos formativos.

La adjudicación e implementación del proyecto Mecesup UAH 0702, durante el año 2009-2010, permitió revisar críticamente lo que se venía realizando, avanzar en un diálogo con otras instituciones y con la política del área y, principalmente, profundizar en los fundamentos, lineamientos y proyecciones de la política institucional sobre "Competencias Habilitantes" $(\mathrm{CH})^{2}$.

2 La reflexión que aquí se presenta es tributaria del trabajo realizado en el marco del proyecto Mecesup UAH 0702 "Diseño de un modelo para el diagnóstico, fortalecimiento y evaluación de competencias para estudiantes de carreras de pedagogía", adjudicado en la línea "Nivelación de competencias básicas para estudiantes desfavorecidos" (2009-2020). 


\section{Dinámicas de ingreso y egreso en educación superior}

\section{Masificación del ingreso}

Uno de los principales cambios que se observa en el sistema de educación superior chileno es el aumento progresivo de su matrícula. En el sistema universitario el acceso se ha extendido a niveles que triplican el número de estudiantes en los últimos 20 años $^{3}$. Ésta es una muy buena noticia: se ha abierto la posibilidad de acceder a programas de formación universitaria a sectores que históricamente estuvieron excluidos. Evidencia de ello es que el año 2009 la tasa de cobertura de la educación superior para jóvenes entre 18 y 24 años fue de $30,8 \%{ }^{4}$ y la matrícula total del sistema universitario, en 2010 , es de 886.884 estudiantes $^{5}$.

Las políticas públicas han estado orientadas a facilitar el acceso, introduciendo mayor equidad en el sistema, lo que permitió lograr las cifras que se han comentado anteriormente. Se trata, principalmente, de ayudas económicas que acercan la posibilidad de costear estudios universitarios que siguen siendo muy onerosos. Becas, créditos, subsidios -públicos y privados- han sido los instrumentos a través de los cuales se ha facilitado el ingreso y la permanencia de los estudiantes con más dificultades económicas ${ }^{6}$. Levantada la compuerta financiera, especialmente a través de la Ley n. ${ }^{\circ} 20.207$, que establece las normas para el financiamiento de estudios en educación superior, promulgada en junio del 2005, el aumento de la demanda fue explosivo. El propio flujo de interesados en ingresar a la universidad hizo caer las otras barreras asociadas a cuestiones académicas: aumento en la oferta de programas, números de vacantes y descenso en los puntajes. ${ }^{7}$

$3 \operatorname{MINEDUC}(2010$, p. 4).

4 Ídem.

5 Datos obtenidos de INDICES (2010), CNED.

6 Sobre el perfil socioeconómico de esta expansión, ver Espinoza, González et al. (2008).

7 Alternativas clásicas como las ingenierías, medicina, derecho o arquitectura, cuyo acceso estaba restringido a grupos relativamente pequeños, venían incrementando su matrícula nacional desde inicios de los 90 con alguna inflexión en los 2000; al igual que otras, menos clásicas, pero tradicionalmente demandadas por el mercado, como psicología o ingeniería comercial. Ver MINEDUC (2010). 
Nos encontramos, entonces, ante un sistema de educación superior que, en su vertiente de estudios universitarios, ha aumentado ostensiblemente sus matrículas, incorporando a un nuevo grupo de estudiantes, que ha sido caracterizado básicamente a través de su perfil socioeconómico.

¿De qué depende que esta importante ampliación de la posibilidad de ingresar a la universidad se transforme en una oportunidad real de formación profesional y académica?

Efectivamente, pensamos que el hecho de ingresar a la universidad no garantiza el acceso a una formación de calidad y conducente a un resultado satisfactorio. Son muchos, múltiples y complejos los factores que incidirán en el éxito de una trayectoria formativa universitaria; entre ellos, comenzaremos por discutir sobre el llamado "perfil de ingreso" de los estudiantes universitarios.

\section{Enriquecer el llamado "perfil de ingreso"}

No basta con una caracterización socioeconómica de los estudiantes que se incorporan al sistema universitario ni tampoco es recomendable atribuir, de modo mecánico, características socioculturales o académicas a dicha condición. Es importante, entonces, ampliar los rasgos a considerar en el "perfil de ingreso"; entre otras cosas, porque en un momento de masificación es crucial que las universidades y sus programas mantengan altas expectativas respecto de los procesos formativos de los estudiantes y de sus potenciales logros, tal como cuando ese ingreso era más selectivo. Esto, con el fin de adecuar la propuesta formativa a los perfiles de ingreso "reales" de los nuevos estudiantes, buscando asegurar una formación universitaria de buen nivel.

Un paso de gran importancia en la dirección de complejizar y enriquecer el análisis de quienes están ingresando a la universidad ha sido el desarrollo de la categoría "primera generación en la educación superior" . Categoría que identifica a aquellos estudiantes que acceden al nivel terciario de la educación, en circunstancias de que sus padres

8 A este respecto, hemos consultado el artículo de Castillo y Cabezas (2010). 
no lograron hacerlo. De acuerdo con los autores citados, el porcentaje de jóvenes que pertenecen a esta categoría representa en la actualidad un $53,5 \%$ en el caso de las mujeres y un $55,8 \%$ en el de los hombres, de la matrícula nueva en la educación superior.

La investigación disponible muestra que la posibilidad de lograr este ingreso, además de contar con nuevas opciones de financiamiento, está asociada a las trayectorias educativas previas, tanto con relación a su extensión (haber asistido a la educación preescolar) como al tipo y dependencia del establecimiento educacional (modalidad científico-humanista y dependencia particular subvencionada) y a los desempeños obtenidos a lo largo de esas trayectorias (notas, repitencia y resultados SIMCE) ${ }^{9}$. Por otra parte, la condición de "primera generación" se vincula al reconocimiento de ciertos déficits socioculturales que pueden llegar a ser significativos al momento de insertarse en el medio universitario y permanecer en él. Un 80\% de quienes desertan de la educación superior serían estudiantes "primera generación"10.

El otro componente estandarizado del perfil de ingreso de los estudiantes universitarios es el puntaje obtenido en sus pruebas de selección (PSU). En su actual modalidad, esta prueba tiene como referencia los contenidos curriculares y los aprendizajes esperados en la educación secundaria, principalmente en términos de habilidades. Se puede discutir sobre su carácter predictivo, pero lo más o menos evidente es que existiría asociación entre los puntajes PSU y niveles de dominio de habilidades básicas en áreas esenciales ${ }^{11}$. En este contexto, resulta ilustrativa la distribución por tramo de puntajes de los estudiantes que rinden la PSU:

9 Castillo y Cabezas, op. cit., p. 70

10 Ídem ant., p. 69.

11 La experiencia institucional de la UAH, al aplicar pruebas de diagnóstico de habilidades básicas a los estudiantes que ingresan, muestra que existe una alta correlación entre los puntajes PSU y los logros en dichas pruebas. Así, por ejemplo, el 33,9\% de los estudiantes que se ubican en el tramo entre 500 y 550 puntos PSU aprueba el diagnóstico en Lenguaje; este porcentaje sube a $50,7 \%$ en los estudiantes entre 551 y 600 puntos, y a un $77,7 \%$, en el caso de aquellos que obtuvieron más de 600 puntos (datos 2010). 
Tabla n. ${ }^{\circ}$ 1: Distribución de estudiantes que rinden la PSU por tramo de puntajes y por años.

\begin{tabular}{|c|c|c|c|c|c|c|c|c|c|}
\hline Año & $\begin{array}{c}\text { Menos } \\
450\end{array}$ & $450-499,5$ & $500-549,5$ & $550-599,5$ & $600-649,5$ & $650-699,5$ & $700-749,5$ & Más de & TOTAL \\
750 & \\
\hline 2010 & 81.736 & 45.269 & 44.413 & 35.894 & 24.369 & 12.330 & 5.445 & 2.178 & 251.634 \\
\hline$\%$ & 32,48 & 17,99 & 17,64 & 14,26 & 9,68 & 4,89 & 2,16 & 0,86 & 100 \\
\hline
\end{tabular}

Fuente: DEMRE. Compendio Estadístico Proceso 2010.

La PSU opera como requisito formal para el ingreso a las universidades chilenas y como filtro al momento de distribuir a los postulantes en las distintas instituciones y carreras; sin embargo, se muestra deficitaria como instrumento de diagnóstico respecto de las habilidades básicas requeridas por los estudiantes universitarios iniciales. Complementar y enriquecer este componente de su perfil supondría -tal como ya lo están haciendo algunas universidadesevaluarlos con mayor precisión en estos ámbitos ${ }^{12}$.

Si bien la necesidad de un diagnóstico más específico puede ser considerada universal respecto del conjunto de estudiantes que ingresan al sistema universitario, se transforma en crítica respecto de aquellos que se sitúan por debajo de... ¿los 600 puntos?, ¿los 550 puntos?, ¿los 500?, en fin, se trata de un umbral que cada institución precisará. Esta información más específica o desagregada permite que los responsables de los procesos formativos universitarios puedan leerla y utilizarla, durante los primeros años, para el fortalecimiento de los estudiantes que lo requieran y puedan así enfrentar con mayor éxito su trayectoria académica universitaria ${ }^{13}$.

\section{¿Más o menos filtros de entrada?}

En un contexto universitario cada vez más consciente de las implicancias de los perfiles de ingreso, ya sea por la comentada masificación de la oferta y del acceso o por el impacto de indicadores de retención o repitencia, se ha instalado la discusión sobre la conveniencia de

12 Por ejemplo, incluir en estas evaluaciones la capacidad de producir textos escritos en el área de lenguaje, o distinguir y cruzar ciertos dominios de contenidos y determinadas habilidades en el campo del pensamiento lógico-matemático.

$13 \mathrm{Al} \mathrm{respecto,} \mathrm{resulta} \mathrm{interesante} \mathrm{el} \mathrm{modo} \mathrm{en} \mathrm{que} \mathrm{Perrenoud} \mathrm{concibe} \mathrm{la} \mathrm{"evaluación} \mathrm{formativa",}$ como "ayuda al alumno a aprender y a desarrollarse". Ver Perrenoud (2008). 
alivianar o de aumentar los filtros de ingreso ${ }^{14}$. Aumento o disminución en los puntajes de corte PSU y/o en la ponderación de las notas de enseñanza media; realización de propedéuticos; bonificaciones de puntaje a los mejores estudiantes de la educación media; incentivos económicos a los mejores puntajes, son todos mecanismos que en la actualidad se utilizan para regular el ingreso de los estudiantes universitarios e intentar atraer a los mejores.

Un caso que ilustra bien esta discusión es la formación inicial docente. Los datos muestran que, mientras en las universidades del CRUCH el puntaje promedio de ingreso a las carreras de pedagogía es 560 puntos, en las universidades privadas es 490 puntos $^{15}$, graficando la diversidad existente e instalando la reflexión sobre el perfil académico necesario de un estudiante que ingresa a la universidad y quiere formarse como profesor o profesora. Frente a esta realidad, el reciente panel de expertos formado por el Gobierno para hacer recomendaciones que mejoren la calidad de la educación sugirió exigir un puntaje mínimo de ingreso superior a 550 puntos y establecer una serie de incentivos económicos para atraer mejores puntajes e incluso puntajes de excelencia ${ }^{16}$.

¿Qué hacer, entonces? ¿Más o menos filtros al ingreso a la formación universitaria? ¿Se privilegia la ampliación de las oportunidades de acceso o se priorizan las perspectivas de éxito académico? Estas disyuntivas tienden a tensionar los aspectos sociales y académicos con relación a los modos y estrategias de democratización de la educación superior universitaria.

Apostar a una alta selectividad en el ingreso tendría efectos regresivos importantes respecto a la ampliación y equidad en el acceso a la educación universitaria, pues como se sabe, los más altos puntajes PSU corresponden en su mayoría a estudiantes de los quintiles más altos. En consecuencia, de mantenerse filtros de selección que sean

14 Más adelante comentaremos también la opción de establecer mayores filtros al egreso o durante la trayectoria formativa.

15 Según el informe final del Panel de Expertos para una Educación de Calidad, disponible en: http://www.mineduc.cl/biblio/documento/201007141115230.Informe\%20final3.pdf

$16 \mathrm{Al}$ respecto ver Latorre (2010). 
razonables y acordes con las características de los egresados de la educación secundaria, independientemente de los incentivos para elevar los puntajes de ingreso, el estudiantado seguirá siendo diverso y una parte importante evidenciará debilidades en las habilidades básicas fundamentales para la educación superior ${ }^{17}$. Éste es, a nuestro juicio, el dato relevante y del cual el sistema universitario debe dar cuenta y hacerse cargo.

Si más allá de su perfil socioeconómico y académico se quisiera mirar a los estudiantes "de frente", sería necesario explorar tanto en sus fragilidades ante las exigencias de los procesos formativos universitarios como en sus potencialidades para avanzar con éxito en ellos. Este diagnóstico posibilitaría identificar con mayor claridad el punto de partida de los procesos formativos y, desde allí, evaluar el tipo de trayectoria que permitiría alcanzar sus perfiles de egreso.

\section{Creciente preocupación por el egreso}

La atención no ha estado puesta sólo en los perfiles de ingreso al sistema universitario; creciente ha sido también la preocupación por los perfiles de egreso y, de modo más general, por los resultados de los procesos formativos, en términos de aprendizajes de salida.

A ello ha contribuido, por una parte, la instalación del mecanismo de acreditación de carreras de pregrado, puesto que a través de sus procesos de autoevaluación han debido formular y/o analizar tales perfiles, la relación de éstos con las mallas curriculares $y$, por último, evidenciar lo que efectivamente aprendieron los estudiantes a lo largo de su formación ${ }^{18}$.

En este contexto, se ha visto reforzada la tendencia a que estos perfiles de egreso estén construidos en lógica de competencias o, por lo menos, en lógica de saberes y capacidades. De este modo, se está dando una señal respecto del tipo de aprendizaje que se espera que logren los estudiantes: se trata de desempeños académicos o

18 Las tres dimensiones sobre las cuales deben versar las autoevaluaciones y los informes de acreditación son: perfil de egreso, condiciones de operación y capacidad de autorregulación. 
profesionales situados en el campo del "hacer". Se observa entonces que, si bien las regulaciones existentes, destinadas a asegurar la calidad de la formación universitaria, no se pronuncian sobre las características que debieran tener los procesos formativos, sí obligan a reflexionar acerca de las estrategias pedagógicas más eficaces para el logro de tales aprendizajes.

Una segunda razón por la cual el momento del egreso ha cobrado relevancia en la discusión es la introducción de pruebas o evaluaciones que buscan verificar lo que las universidades y sus programas están certificando con sus grados y títulos. Es el caso de las pruebas nacionales para los egresados de las carreras de medicina y de pedagogía: el Examen Único Nacional de Conocimientos de Medicina ${ }^{19}$ y la Evaluación Diagnóstica Inicia ${ }^{20}$. En el primero de los casos, el examen va más allá de la verificación de logros del proceso formativo y opera también como habilitación laboral. Este tipo de mecanismos e instrumentos, aunque puede discutirse respecto de la oportunidad de sus resultados, ponen una presión importante sobre el momento del egreso: se faculta a un tercero -distinto a cada una de las universidades- para que determine si los perfiles de egreso están o no logrados.

Por último, el momento del egreso se ha constituido en un factor relevante por su incidencia en la llamada "tasa de titulación oportuna", que indica la proporción de estudiantes que logran terminar sus estudios de pregrado en el tiempo previsto por el plan de estudios más un margen de un año. Vale decir la calidad de la formación no sólo se mide por los aprendizajes que los estudiantes lograrían al finalizar su proceso formativo, sino también por la oportunidad en que éstos se alcanzarían.

19 El Examen Único Nacional de Conocimientos de Medicina (EUNACOM) está destinado a evaluar los conocimientos de los médicos que inician su ejercicio profesional en el país. Regulado por la Ley 20.261, el examen es administrado por la Asociación de Facultades de Medicina de Chile (ASOFAMECh). Disponible en: http://www.eunacom.cl/

20 El objetivo central de la Evaluación Inicia es entregar a las instituciones formadoras de profesores información actualizada acerca del nivel de logro de sus estudiantes egresados en aspectos fundamentales del futuro desempeño de éstos como docentes. El Programa Inicia, además de este componente de evaluación, incluye otros dos: Orientaciones curriculares y Programa de apoyo a instituciones. Disponible en: http://www.programainicia.cl/ed01.html 
Según los datos del informe sobre educación superior de la OCDE y el Banco Mundial del año 2009, la tasa general de titulación en las universidades chilenas es baja: entre 1998 y 2002 en promedio alcanzó un 46,3\%. Ello abre interrogantes no sólo sobre la viabilidad de los perfiles de egreso, sino también sobre la adecuación de los perfiles de ingreso a las exigencias del proceso formativo $y$, tanto o más importante, sobre las trayectorias formativas en cuanto expresión del tránsito entre perfil de ingreso y perfil de egreso ${ }^{21}$.

En suma, la relevancia que ha adquirido el momento del egreso de los procesos formativos universitarios permite constituir, como objeto de estudio y de reflexión, pero sobre todo como campo de acción, el espacio de tránsito entre las condiciones de ingreso y las de egreso. Más aun, en un contexto en que, tal como se ha señalado anteriormente, los perfiles de ingreso de los estudiantes universitarios se han visto fuertemente modificados. Ese espacio de tránsito, representado en el siguiente diagrama, es el que denominaremos - para efectos de este artículo- "trayectoria formativa universitaria"22.

Gráfico n. ${ }^{\circ} 1$

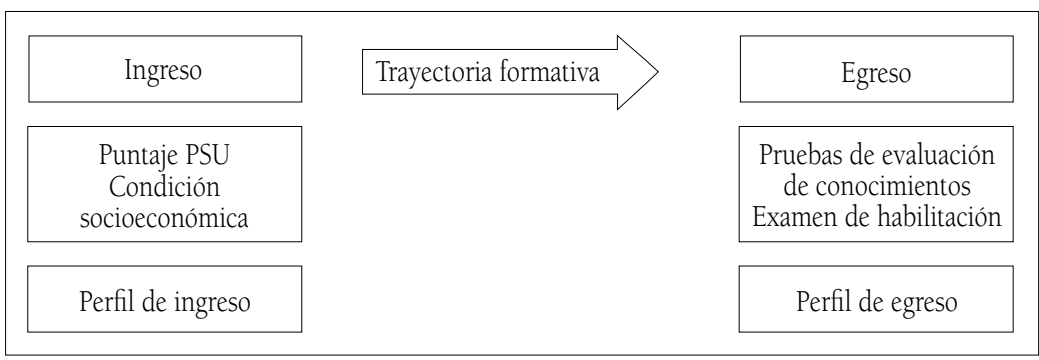

Fuente: elaboración propia.

21 OCDE y Banco Internacional para la Reconstrucción y el Desarrollo / Banco Mundial (2009).

22 Habitualmente, el término "trayectoria" es utilizado para referirse a las posiciones sociales ocupadas por un grupo o individuo en un campo determinado. En este caso, lo restringiremos al recorrido de los estudiantes durante sus programas de formación universitaria; y entenderemos por trayectoria, entonces, tanto la propuesta formativa como la experiencia de formación vivida por los estudiantes en el marco de esa propuesta. Este concepto se puede vincular también a la noción de "itinerario formativo", siendo la trayectoria, en este caso, la manera concreta en que un grupo o un individuo experimenta dicho itinerario. 


\section{Competencias habilitantes: una apuesta por fortalecer las trayectorias formativas universitarias}

\section{La centralidad de las trayectorias formativas}

Si las premisas anteriores -el impacto de la ampliación del acceso a la educación universitaria y la necesidad de traducirlo en oportunidad real de formarse profesional y académicamente- son correctas, resulta ineludible interrogarse acerca de la capacidad de las trayectorias formativas universitarias para hacer transitar a sus estudiantes desde las características de sus perfiles de ingreso hacia perfiles de egreso crecientemente exigentes.

Durante las trayectorias formativas universitarias los estudiantes ponen en juego sus conocimientos previos y sus capacidades de aprender en el contexto de propuestas curriculares o planes de estudios, que definen tanto los aprendizajes a lograr como el tiempo estimado para ello (tiempos parciales y tiempos agregados). El propósito principal de una trayectoria formativa debiese ser el logro del perfil de egreso asociado a una propuesta curricular o plan de estudios; por lo tanto, una trayectoria formativa se habrá cumplido exitosamente en la medida que el estudiante desarrolle las competencias contenidas en dicho perfil, en los tiempos programados. Esta adecuación, en el contexto del Sistema de Aseguramiento de la Calidad, se mide a través de la "tasa de titulación oportuna".

Si bien las trayectorias formativas son diseños o propuestas que responden a una generalización, en la práctica son recorridos individuales. Ambas dimensiones -la general y la individual- obligan a considerar los perfiles de ingreso de los estudiantes que inician una determinada trayectoria formativa. En el caso del diseño general de la propuesta, caracterizar el "estudiante tipo" respecto del cual se formula la hipótesis formativa es fundamental, y para el caso de la apropiación individual que cada sujeto hace de la propuesta, será clave contar con el máximo de antecedentes sobre sus conocimientos disciplinares y habilidades previas.

De la naturaleza de los perfiles de egreso dependerán también las características de las experiencias de aprendizaje que conduzcan 
a su logro. Si el perfil de egreso está formulado, por ejemplo, en clave de competencias, como hemos visto que es la tendencia actual, la propuesta curricular o plan de estudios deberá contemplar experiencias de aprendizaje que permitan efectivamente el desarrollo de competencias. Sobre este punto y la viabilidad o conveniencia de un enfoque "mixto" volveremos un poco más adelante.

Las "mallas curriculares" pueden ser entendidas como el referente más habitual al momento de pensar en trayectorias formativas. Son, básicamente, la formalización gráfica de la articulación y distribución en el tiempo de las distintas actividades formativas que -se postulaconducirán al logro de un determinado perfil de egreso. Ellas explicitan el itinerario formativo que debiera seguir un sujeto para alcanzar el perfil de egreso propuesto. Se trata, sin embargo, de una representación que cobra su pleno sentido al formar parte de un "plan de estudios" que explicita las condiciones de su implementación. Así, por ejemplo, el plan de estudios puede definir áreas o líneas de formación y/o ciclos, si los hubiera, y sus fundamentos; la existencia o no de prerrequisitos entre actividades formativas; los requisitos de ingreso y de egreso; la articulación entre actividades teóricas y prácticas si las hubiese; en fin, todo aquello que, en términos curriculares, orienta e incide en el proceso formativo que se propone.

Las trayectorias formativas están lejos, entonces, de ser algo así como un funicular, que conduce mecánicamente desde una estación de partida hasta una estación de llegada. Las trayectorias formativas son hipótesis generales que deben demostrarse en la práctica; no son lineales ni tienen su éxito asegurado, ya que son vivenciadas individualmente por sujetos distintos con diversos tipos de motivación y provistos de recursos muy variados.

En consecuencia, las trayectorias formativas universitarias son recorridos que deben ser monitoreados y evaluados permanentemente. Lo más habitual ha sido colocar las instancias de evaluación al final de los recorridos, al momento del egreso y de la obtención de los títulos y/o grados. Aunque, crecientemente, hay iniciativas que han desarrollado evaluaciones diagnósticas al inicio de las trayectorias, con el fin-como ya hemos comentado- de contar con información 
más precisa sobre los perfiles de ingreso. Surge, en este contexto, la pregunta por las evaluaciones intermedias. ¿Basta con la evaluación de las actividades formativas, una a una, en el contexto de un plan de estudios, para asegurar el avance de los aprendizajes esperados? ¿Es el logro de los perfiles de egreso el resultado de la simple adición de dichos aprendizajes?

\section{Aprendizajes progresivos e integrados}

La introducción de la "lógica" de competencias en la educación superior, principalmente respecto de perfiles de egreso, ha llevado a la pregunta por las características que debiera tener un proceso formativo que conduzca al desarrollo de competencias ${ }^{23}$.

Existe, por una parte, una respuesta clásica que se relaciona con el llamado "modelo curricular basado en competencias", que propone considerar las competencias como unidades a partir de las cuales estructurar el currículum más que como puntos de llegada en términos de aprendizaje ${ }^{24}$. De allí que en este tipo de modelo "más ortodoxo", por ejemplo, se proceda a la modularización de las actividades formativas en función de las competencias que se quiere desarrollar, se opte por metodologías que integren las actividades teóricas y prácticas o se cuente con sistemas para la certificación de unidades de competencias.

Sin embargo, por otra parte y como resultado del análisis crítico de las experiencias clásicas, han surgido propuestas formativas menos radicales, que postulan un currículum orientado al desarrollo de competencias. Se trata de un modelo que en términos curriculares no estructura sus planes de estudio ni sus mallas a partir de competencias; no modulariza los itinerarios ni renuncia, por ejemplo, a los cursos o

23 Escapa al propósito de este artículo profundizar en las conceptualizaciones y discusiones que se han desarrollado en torno al concepto de competencias y el modo en que los sistemas de educación superior lo han asumido. Existe al respecto abundante bibliografía, entre la que se puede mencionar: Villa y Poblete (2007), Tobón (2010), Jonnaert (1999).

24 Marie Françoise Legendre, académica canadiense de la Universidad Laval, expresa bien esta distinción, tal como lo plantea Joan Pagès: "(ella) establece una diferencia entre la competencia como principio organizador de los planes de estudio o de la metodología de elaboración de los currículos, y la competencia como una visión renovada del aprendizaje..." (Pagès, 2009, p. 30). 
a las asignaturas como modos de organizar las actividades formativas, ni tampoco a la separación entre actividades teóricas y prácticas. Pero sí interroga el currículum desde la pregunta por su contribución al desarrollo de las competencias que se busca desarrollar en los estudiantes. Es decir, una perspectiva que valoriza la noción de competencia como una manera particular de entender los aprendizajes, pero que deja abiertas las posibilidades pedagógicas y didácticas para lograr dichos aprendizajes. Éste es un tipo de modelo que puede denominarse "mixto", en el sentido de que asume las competencias como una nueva forma de concebir los aprendizajes, pero no necesariamente como principios organizadores del currículum ${ }^{25}$.

De cualquier modo, con independencia del modelo pedagógico que se adopte, lo insoslayable de un enfoque "por" u "orientado a" competencias es la manera de entender los aprendizajes: un sujeto aprende, o sea es competente, cuando es capaz de enfrentar y resolver una situación-problema movilizando de modo integrado una serie de recursos de los cuales dispone (cognitivos, sociales, afectivos, entre otros $)^{26}$. En esta perspectiva, la capacidad del sujeto para movilizar de modo integrado sus conocimientos es clave. Por lo tanto, para "aprender", no basta con poseer o adquirir los conocimientos, es necesario poder "hacer algo" con ellos, movilizarlos e integrarlos en función de la naturaleza de la situación-problema que se busque enfrentar o resolver. La literatura sobre competencias, ya tiene más o menos formalizados y consensuados tales conocimientos y saberes:

25 Otra manera de abordar la distinción entre "formación basada en competencias" y "formación orientada hacia la competencia" es la siguiente:

"En Alemania, el concepto de competencia se utiliza para definir la integración y articulación de capacidades, habilidades y conocimientos en la estructura profunda de la personalidad que permiten al individuo la solución y anticipación de problemas complejos en contextos distintos. En este sentido, el objetivo del diseño curricular es estructurar procesos integrales de enseñanza-aprendizaje cuyo diseño metodológico-didáctico oriente al individuo hacia la adquisición de competencias y le posibilite, de esta manera, desenvolverse en distintas áreas de desempeño (kompetenzorientiertes Lernen). En este sentido, se distingue del competency based training británico, que concibe la competencia profesional como un agregado de competencias certificadas, relacionadas con distintas funciones ocupacionales y/o profesionales". Hans-Jürgen Lindemann (Buenos Aires) / Rudolf Tippelt (Munich), Competencias clave y capacidades profesionales básicas - una selección de aspectos y fundamentos, disponible en: http://www.halinco.de/html/doces/TIPP-LI-Compet-clave0799.pdf

26 Esta manera de entender las competencias ha sido ampliamente desarrollada por autores francófonos tanto europeos como canadienses. Para una aproximación que sintetiza bien esta perspectiva, ver Roegiers (2007). 
conceptuales, procedimentales y actitudinales o, dicho de otro modo, conceptos o contenidos disciplinares; capacidades y habilidades; y actitudes.

Teórica y metodológicamente, la perspectiva socioconstructivista de las competencias, desarrollada y sistematizada por Jonnaert (2002), proporciona distinciones conceptuales útiles para sustentar el análisis que proponemos. En efecto, utilizando la metáfora de las "muñecas rusas", Jonnaert sostiene que entre los muchos recursos que una competencia debe convocar para desplegarse, están las capacidades, las que, a su vez, para su concreción requieren movilizar habilidades, todo ello, a partir de una situación-problema, que es la que demanda el despliegue de la competencia. Las capacidades son entendidas, principalmente, como recursos cognitivos; y las habilidades, en general, como recursos cognitivo-motrices.

En esta perspectiva, las competencias no son sólo cognitivas; se inscriben en situaciones que tienen dimensiones contextuales y dimensiones afectivas y sociales. De allí que no puedan considerarse sólo como entidades cognitivas descontextualizadas y deban ser "situadas". Sin embargo, una o varias capacidades cognitivas pueden ser constitutivas o estar a la base de una competencia. En esta conceptualización, las capacidades refieren a una formación general, común a muchas situaciones, en tanto que una competencia remite más bien a una formación más específica, que pone en juego, normalmente, varias capacidades en una misma situación.

Para Jonnaert, el concepto de capacidad remite a conocimientos reproducibles en diversos campos; a conocimientos transversales, utilizables por el sujeto en distintas situaciones. Para que una competencia las pueda convocar, ellas (las capacidades) deben estar estabilizadas. Habilidad, en cambio, es un término más específico que capacidad y se centra en una tarea fácilmente identificable. La descripción de una habilidad comprende una acción (física o intelectual) y el contenido disciplinar al cual se refiere la acción; por ejemplo, producir textos escritos de tipo argumentativo. Normalmente las habilidades no pueden funcionar si no son convocadas por una capacidad, la que, a su vez, funciona en la puesta en práctica de 
una competencia en situación. De ese modo se termina de graficar la evocación de las "muñecas rusas". De acuerdo con Jonnaert, capacidades, habilidades y contenidos disciplinares es una tríada que, a su vez, sirve de esqueleto a la competencia, que la moviliza junto a otros recursos. Se trata, a su juicio, de una movilización "en cascada"...27.

Las trayectorias formativas universitarias que se orienten al desarrollo de competencias, en consecuencia, debieran promover procesos de aprendizaje progresivos e integrados (sin los cuales, no hay "cascada"...). Esta necesidad es más clara y evidente en aquellas propuestas o modelos que no basan su currículum en competencias, es decir, aquellos que hemos denominado como mixtos. Esto, justamente porque esa mixtura permite, en paralelo, la adquisición o desarrollo de los recursos que concurrirán a las competencias a lograr (capacidades, habilidades y contenidos disciplinares), admitiendo una diversidad de estrategias didácticas. Sin embargo, el proceso o trayectoria formativa debiera prever momentos en que esos aprendizajes "parciales" puedan ser integrados, para dar paso así, progresivamente, a aprendizajes más complejos, conducentes al desarrollo de competencias. En esta lógica, los planes de estudio deben ir resolviendo la ecuación entre: (a) el reforzamiento, en los semestres o ciclos iniciales, de capacidades y habilidades básicas comunes para cualquier estudiante universitario, necesarias para construir aprendizajes crecientemente complejos, y b) el desarrollo progresivo de los recursos propios de los ciclos de especialización, que permitirán alcanzar las competencias profesionales de cada carrera. Esta progresión que combina y pondera el reforzamiento de capacidades y habilidades básicas ${ }^{28}$ y el desarrollo de competencias profesionales ${ }^{29}$ a lo largo de los años de formación puede ser graficada del siguiente modo:

27 Una versión más reciente en la cual Jonnaert trabaja las implicancias curriculares de la perspectiva socioconstructivista de las competencias, en Jonnaert (2008).

28 Aquellas capacidades y habilidades básicas y genéricas que los estudiantes debieran poseer al inicio de su vida universitaria teniendo como referentes los aprendizajes esperados al término de la enseñanza secundaria. En tanto básicas y genéricas, movilizables en función de diversas competencias.

29 Aquellas competencias contenidas en el perfil de egreso de cada una de las carreras, que suponen la movilización tanto de capacidades y habilidades como de contenidos disciplinares específicos y que debieran corresponder a las competencias de un profesional novel. 
Gráfico n. ${ }^{\circ} 2$

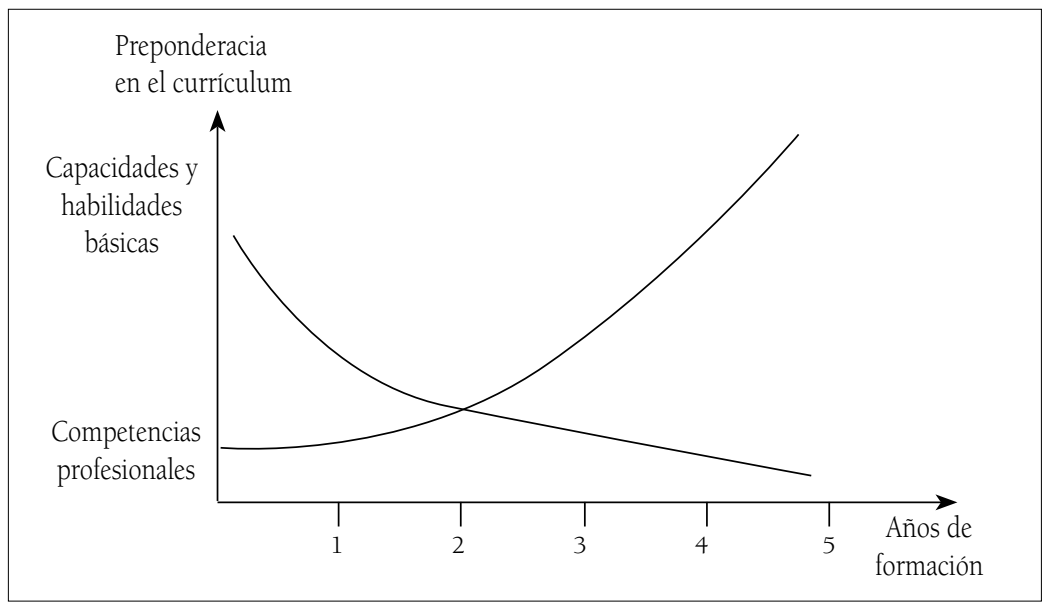

Fuente: elaboración propia.

Así, durante los primeros años de formación, los estudiantes debieran fortalecer y ejercitarse en la movilización de sus capacidades y habilidades básicas en función -a medida que el proceso formativo avanza- de la adquisición de conocimientos conceptuales y desarrollo de capacidades y habilidades más específicas, que remiten a la formación profesional y académica propia de su campo disciplinar.

En consecuencia, estos aprendizajes, que hemos caracterizado como progresivos e integrados, no sólo son necesarios de cara al logro de los perfiles de egreso (terminales) de un programa de formación o carrera, sino también como aprendizajes que habilitan a los estudiantes para recorrer exitosamente sus trayectorias formativas. Vale decir, aprendizajes para continuar aprendiendo, de acuerdo con las exigencias de los distintos niveles o ciclos de formación.

\section{La noción de competencias habilitantes}

En el contexto descrito, y tal como lo hemos señalado, la necesidad de asegurar el progreso de los aprendizajes de los estudiantes durante la formación inicial es una tarea fundamental. No sólo en la perspectiva de monitorear cuantitativa y formalmente los avances de cada cohorte en su trayectoria, sino en la idea de levantar información que permita identificar quiénes van avanzando, en qué condiciones y quiénes 
no lo hacen. Esta preocupación instala la necesidad de interrogarse, oportunamente, sobre los aprendizajes que los estudiantes alcanzan no solo al término de su trayectoria, sino como medios para realizar dicho tránsito de manera exitosa.

¿Hasta qué punto los planes de estudios consideran aprendizajes claves que habiliten a los estudiantes para desarrollar al máximo sus capacidades, en función de lograr los perfiles de egreso? ¿Es posible identificar y evaluar esos aprendizajes? Es a este tipo de interrogantes que busca contribuir la noción de "competencias habilitantes", entendida como aquellos aprendizajes que los estudiantes debieran poseer al cabo de sus primeros años de formación universitaria, útiles para enfrentar de manera más exitosa las oportunidades de seguir aprendiendo, que les ofrecen los ciclos de formación profesional de sus carreras específicas ${ }^{30}$. ¿Qué requiere haber aprendido -en clave de competencias, es decir, haber adquirido y saber utilizar de modo integrado en situación- un estudiante a fines del segundo año, por ejemplo de Licenciatura en Historia, para poder continuar con mayor éxito su formación? ¿Qué lo diferenciaría de un estudiante de Psicología o Derecho? Los requerimientos, ciertamente, son distintos,

30 En la literatura sobre competencias, la noción de "competencias habilitantes" no es de uso frecuente y cuando se utiliza, se lo hace de modo genérico, para referirse a aquello para lo cual una competencia habilita (por ejemplo, "competencia habilitante para tal desempeño"). El sentido con el cual la hemos utilizado en la Universidad Alberto Hurtado es más específico y está menos desarrollado en la bibliografía sobre competencias. Una de las definiciones que se acerca al modo como la proponemos, pero que está referida a las llamadas competencias fundamentales, es la siguiente: "llamaremos 'competencias fundamentales' a aquellas competencias que se encuentran en la base de todas las áreas ocupacionales, aunque su forma específica o nivel de complejidad puede variar según el área. Son las habilidades que la gente utiliza para llevar adelante una amplia variedad de tareas de la vida diaria y de sus ámbitos laborales. No son habilidades técnicas requeridas por una ocupación, más bien son requeridas por todas las ocupaciones, aunque de modos específicos. Por ejemplo, la escritura es una habilidad o competencia requerida por un amplio rango de ocupaciones, pero la frecuencia y complejidad de su uso varía según la ocupación específica.

Son competencias que habilitan a los sujetos para aprender técnicas específicas para el desarrollo de determinadas tareas. Por ejemplo un reparador puede recibir instrucciones a través de órdenes de trabajo escritas, que ellos deben leer y comprender antes de poder resolver las reparaciones que deban realizarse.

(...) En suma, las competencias esenciales o fundamentales son competencias habilitantes, en tanto sustentan y son necesarias para el desarrollo de las competencias específicas de un perfil profesional". Estela Silvia Barba: Competencias fundamentales, la experiencia canadiense sobre perfiles ocupacionales por competencias fundamentales, disponible en: http://www.halinco.de/html/doces/TIPP-LI-Compet-clave0799.pdf. El subrayado es de los autores. 
aunque es probable que todos ellos comprometan más o menos las mismas capacidades y habilidades básicas.

Las competencias habilitantes $(\mathrm{CH})$, entonces, aluden a competencias que movilizan e integran capacidades y habilidades que poseen (o debieran poseer) los estudiantes en áreas básicas fundamentales y conocimientos -generales y disciplinares- logrados en los primeros años de su formación de pregrado. Competencias que evidencian desempeños consistentes con lo esperable, de acuerdo con el momento de la trayectoria formativa en que se encuentren, y que los "habilitan" para proseguir convenientemente sus estudios. De este modo, pueden ser entendidas también como competencias básicas aplicadas a campos de formación específicos.

En esta perspectiva, los aprendizajes con que llegan los estudiantes a primer año de universidad son importantes y requieren ser conocidos por la institución y sus actores, pues constituyen el punto de partida de los itinerarios de formación que emprenderán. Tal como fuera señalado precedentemente, los puntajes de la PSU son considerados como un referente estandarizado, útil como filtro de entrada o simplemente como requisito formal, aunque insuficiente para cualificar tal punto de partida. En el ámbito de estas preocupaciones, se ubican las iniciativas de algunas universidades por diagnosticar de manera más precisa las habilidades básicas de entrada de sus estudiantes, principalmente en áreas tales como comunicación escrita, razonamiento lógico-matemático, entre otras ${ }^{31}$. Sabido es que la educación secundaria en el país muestra dificultades para asegurar que sus egresados las posean y que, dependiendo de la postura de las instituciones ante el tema de los filtros de entrada, muchas universidades optan por hacerse cargo de este déficit, otras por seleccionar, y algunas, simplemente, omiten esta realidad. El realismo y viabilidad de la opción por asumir las carencias dependerá, en gran medida, de la calidad y precisión del diagnóstico inicial.

Identificar las $\mathrm{CH}$ en el marco de una trayectoria formativa tensiona los planes de estudios en su implementación, llamando la

31 En general se trata de pruebas o tests que efectivamente evalúan "habilidades" o "capacidades", y no necesariamente competencias. 
atención acerca de las características de las actividades curriculares contenidas en los primeros años de formación universitaria; interpelándolas respecto de propósitos formativos más inmediatos que los perfiles de egreso, que tienen un carácter terminal. De este modo, se puede intencionar su contribución al logro de los aprendizajes esperados en los primeros años de formación. Establecer las $\mathrm{CH}$ supone establecer un diálogo entre los ciclos de formación, ya que implica reconocer cuáles son las principales exigencias que enfrentará un estudiante durante su ciclo de formación específica disciplinar y/o profesional. Los docentes y equipos responsables de cada carrera son actores clave para indicar el nivel de aprendizaje esperado de sus estudiantes, traduciendo esto en un referencial de competencias intermedias o habilitantes.

\section{Perfiles intermedios}

Las $\mathrm{CH}$, en consecuencia, permiten formular o explicitar un perfil de nivel intermedio que cumpliría la función de articular capacidades y habilidades en áreas básicas con el desarrollo de competencias disciplinares específicas de una carrera ${ }^{32}$. Se trata de aprendizajes integrados que se espera que sean alcanzados a medio camino de la trayectoria de formación. Su logro requiere ser evaluado en el contexto de situaciones preparatorias a la formación profesional que el estudiante deberá enfrentar durante el ciclo de especialización de su carrera. Si retomamos el gráfico n. ${ }^{\circ} 1$, la representación de este perfil intermedio sería la siguiente:

32 ¿Perfil intermedio o perfil de competencias intermedias? La verdad es que en la literatura sobre competencias existe la definición de intermedias o generativas, para referirse a aquellas capacidades que permiten profundizar o adquirir otras habilidades, como parte de un proceso encadenado de aprendizajes (ver: Guzmán, Ronald. Hacia una Educación Profesional Minera por Competencias, disponible en: http://www.areaminera.com/Contenidos/ Opinion/ronaldg/35.act). En el caso de nuestra propuesta, hemos optado por la noción de "perfil intermedio" para enfatizar que las "competencias habilitantes" no constituyen, necesariamente un estadio de desarrollo de las competencias terminales contenidas en el perfil de egreso, sino un referencial de competencias para seguir aprendiendo, es decir, que tiene como foco la trayectoria formativa. 
Gráfico n. ${ }^{0} 3$

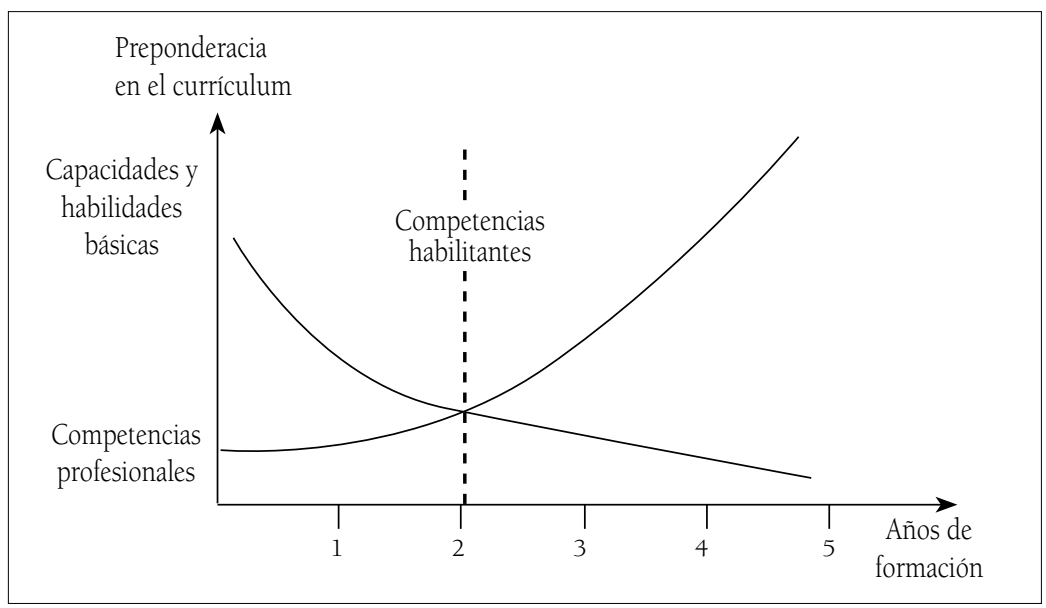

Fuente: elaboración propia.

Si se actúa bajo el supuesto de que la hipótesis formativa contenida en el plan de estudios de la carrera funciona y de que ingresa un "estudiante tipo" con un "perfil tipo" de capacidades y habilidades ya logradas, la formulación de este perfil intermedio puede aparecer como una tarea relativamente fácil. Sin embargo, si se reconoce que los estudiantes que ingresan al sistema universitario no responden a ese perfil ideal ni poseen -en términos de cohorteun perfil homogéneo, se introduce un componente de varianza que pone en tensión el cumplimiento de la propuesta formativa en las condiciones inicialmente definidas (de rendimiento y tiempo). En tal sentido, incorporar la noción de $\mathrm{CH}$ implica definir qué aprendizajes deben lograr aquellos estudiantes "reales" -que ingresan a una carrera específica- durante los primeros años de su formación.

Reconociendo que este perfil intermedio está formulado en términos de "competencias", es necesario introducir en los planes de estudio instancias de evaluación más complejas que los mecanismos de evaluación por asignaturas o cursos. En tanto "competencias", las $\mathrm{CH}$ requieren ser evaluadas a través de "situaciones de integración" en las que los estudiantes demostrarán su capacidad de enfrentarlas y resolverlas, movilizando recursos personales y otros adquiridos en su formación. 
El perfil intermedio de $\mathrm{CH}$ permite verificar qué han aprendido los estudiantes durante los primeros años de su formación, y a la vez identificar qué es aquello que no han aprendido y que constituye condición para seguir adelante. De ese modo, podrán generarse estrategias de fortalecimiento pertinentes a los requerimientos de la trayectoria formativa ${ }^{33}$. Esto implica realizar acciones ya no para nivelar al estudiante o remediar aquello que no tiene, sino para fortalecer aprendizajes más complejos e integrados que aún están insuficientemente desarrollados.

En el caso de aquellos estudiantes que no cumplieran satisfactoriamente con el perfil intermedio, se debiera prever la realización de actividades curriculares complementarias o el desarrollo de instancias de autoformación en los ámbitos específicos pertinentes. En cualquier caso, lo central es fortalecer oportunamente y dentro del currículum el logro de aquellos aprendizajes que, aunque introductorios, sean centrales para avanzar en su carrera.

En síntesis, las $\mathrm{CH}$ constituyen un recurso al servicio del desarrollo de trayectorias formativas más exitosas. En efecto, el interés y el carácter innovador del concepto radican en que compromete no las competencias profesionales a lograr al final de la carrera, sino más bien, las competencias necesarias para el éxito en el recorrido formativo en los campos específicos de formación.

\section{Comentarios finales}

La masificación del ingreso a la educación superior, básicamente por la ampliación del subsidio a la demanda, trajo consigo nuevos desafíos para las instituciones universitarias. Frente a un perfil de ingreso cambiante y heterogéneo, si queremos continuar manteniendo altos estándares de calidad para la formación, se hace ineludible la reflexión continua sobre los procesos educativos. Situarse desde una perspectiva que pone el foco en las trayectorias formativas, promovería una comprensión más amplia y compleja de este proceso, así como

33 Una distinción pertinente a este respecto es la establecida por De Ketele, quien habla de "nivel básico" o de umbral para referirse a aprendizajes de entrada a otros más complejos. Ver De Ketele (1996). 
de las dinámicas sociales involucradas en él. Esta alternativa permite, además, tensionar las hipótesis de la formación inicial, no sólo desde una perspectiva curricular o pedagógica universitaria, sino también política y estratégica. Esto cobra relevancia, especialmente, en un contexto en que la competitividad del sistema educativo no garantiza la calidad del servicio que se ofrece. Esto ha dado origen a un legítimo debate acerca de la calidad de las universidades, tanto públicas como privadas, poniendo en cuestión las características de la formación que reciben sus estudiantes y la calidad de sus resultados finales, expresados en el logro efectivo de sus perfiles de egreso.

En el marco de esta situación y aspirando a perfiles de egreso cada vez más exigentes, resulta fundamental transparentar y monitorear cada una de las etapas que estructuran las trayectorias formativas. En primer lugar, porque disponer de más información acerca de las habilidades y capacidades que poseen quienes ingresan a la universidad permite cualificar tempranamente sus condiciones de entrada a una trayectoria formativa específica. Las instituciones y sus carreras contarían así con información adicional útil no necesariamente para cambiar lo proyectado, sino para adecuar aspectos de sus diseños; posibilitando la integración de mecanismos y dispositivos de fortalecimiento necesarios para que los estudiantes realicen un proceso formativo de calidad y acorde con las características de su grupo en particular.

En segundo lugar, porque la introducción de la noción de competencias habilitantes, que identifica los aprendizajes que los estudiantes de una carrera determinada debieran poseer al cabo de sus primeros años de formación, permite definir una meta a medio camino. Ello implica la formulación de un perfil intermedio que permita a cada carrera imaginar una situación que demande la integración de habilidades básicas, saberes y conocimientos disciplinares; incorporar más oportunamente los reforzamientos necesarios, y por último, tomar decisiones respecto a qué iniciativas seguir si sus estudiantes no están logrando dicho perfil. Las acciones y consecuencias de este perfil no serán únicamente para los estudiantes; serán también para la carrera, pues estas evidencias podrían implicar que la hipótesis formativa requiere de ajustes en su diseño y/o en su implementación. 
Por último, la incorporación de estrategias remediales fuera del currículum se ha mostrado poco eficiente; instalar una nivelación de manera muy temprana es tarea propia de un propedéutico, y reforzar hacia el término de la trayectoria puede ser tardío y con consecuencias de tiempo y costo, básicamente para los estudiantes. Optar, en cambio, por colocar el acento en las trayectorias formativas e incorporar la noción de competencias habilitantes favorece una gestión curricular más oportuna y efectiva para que, finalmente, los perfiles de egreso sean alcanzados de manera exitosa por todos los estudiantes, cualesquiera sean sus puntos de partida, y en un tiempo razonable.

\section{Referencias bibliográficas}

Barba, E. (2009) Competencias fundamentales, la experiencia canadiense sobre perfiles ocupacionales por competencias fundamentales, en: http:// www.halinco.de/html/doces/TIPP-LI-Compet-clave0799.pdf

Castillo, J. y Cabezas, G. (2010) Caracterización de jóvenes primera generación en educación superior. Nuevas trayectorias hacia la equidad educativa, en: revista Calidad en la Educación, (32), julio, pp. 43-76.

De Ketele J.M. (1996) L'évaluation des acquis scolaires: quoi? Pourquoi? Pour qui?, en: Revue Tunisienne des Sciences de l'Education, (23), pp. 17-36.

Espinoza, O.; González, L.E. et al. (2008) Perfil socioeconómico del estudiantado que accede a la Educación Superior en Chile (1990-2003), en Revista Estudios Pedagógicos, XXXIV, (1), pp. 27-39.

Gallardo, G. y Reyes, P. (2010) Relación profesor-alumno en la universidad: arista fundamental para el aprendizaje, en: revista Calidad en la Educación (32), julio 2010, pp. 77-108.

Guzmán, R., Hacia una Educación Profesional Minera por Competencias, en: http://www.areaminera.com/Contenidos/Opinion/ronaldg/35.act

INDICES (2010) Proceso de matrícula 2010. Matrícula primer año y total; Consejo Nacional de Educación.

Informe final del Panel de Expertos para una Educación de Calidad, http:// www.mineduc.cl/biblio/documento/201007141115230.Informe\%20 final3.pdf 
300 COMPETENCIAS HABILITANTES: UN APORTE PARA EL REFORZAMIENTO DE LAS TRAYECTORIAS FORMATIVAS UNIVERSITARIAS - Marisol Latorre, Paloma Aravena, Pedro Milos, Miguel García

Jonnaert, Ph. (1999) Créer des conditions d'apprentissage. Un cadre de référence socio constructiviste pour une formation didactique des enseignants, De Boeck, Paris-Bruxelles.

Jonnaert, Ph. (2002) Compétences et socioconstructivisme. Un cadre théorique, De Boeck, Bruselas.

Jonnaert, Ph. (2008) Logique de compétences et développement curriculaire, L'Harmattan, Paris.

Lindemann H. J. (Buenos Aires) / Tippelt, Rudolf (Munich), Competencias clave y capacidades profesionales básicas, una selección de aspectos y fundamentos, en: http://www.halinco.de/html/doces/TIPP-LI-Competclave0799.pdf

Latorre, M. (2010) Notas para la Discusión sobre profesionalización docente, en: Cuadernos de Educación, (26), septiembre, Facultad de Educación, Universidad Alberto Hurtado, disponible en: www.educación. uahurtado.cl

MINEDUC (2010) Compendio Estadístico Proceso de Admisión Año Académico.

MINEDUC (2010) Evolución de la matrícula de Educación Superior en Chile. Periodo 1990-2009, SIES, División de Educación Superior, junio.

http://www.eunacom.cl/

http://www.programainicia.cl/ed01.html

Ministerio de Educación (2009) Los libros de texto de ciencias sociales, geografía e historia y el desarrollo de competencias ciudadanas, en: Seminario Internacional Textos Escolares de Historia y Ciencias Sociales; Santiago de Chile, 2008.

OECD y Banco Internacional para la Reconstrucción y el Desarrollo / Banco Mundial (2009) La Educación Superior en Chile.

Pagès, J. (2009) Los libros de texto de ciencias sociales, geografía e historia y el desarrollo de competencias ciudadanas, en: Seminario Internacional Textos Escolares de Historia y Ciencias Sociales; Santiago de Chile, 2008, Ministerio de Educación.

Perrenoud, Ph. (2008) La evaluación de los alumnos: de la producción de la excelencia a la regulación de los aprendizajes; Colihue, Buenos Aires.

Roegiers, X. (2007) Pedagogía de la Integración. Competencias e integración de los conocimientos en la enseñanza; Coordinación Educativa y Cultural Centroamericana, San José de Costa Rica.

Tobón, S. (2010) Formación integral y competencias. Pensamiento complejo, currículo, didáctica y evaluación, ECOE Ediciones, Bogotá. 
UAH-CIDE (2006) Encuesta a Actores Educativos, disponible en: www.cide.cl Villa, A. y Poblete, M. (directores) (2007) Aprendizaje basado en competencias. Una propuesta para la evaluación de las competencias genéricas; Ediciones Mensajero, Bilbao.

Recibido: 27/09/2010

Aceptado: 14/11/2010 